\title{
Adherence to Exercise Training in COPD Patients- Factors that Predict Patient Adherence and Non-Adherence- A Preliminary Study
}

\author{
Renukadevi Mahadevan ${ }^{1}$, Chaya S.K². Pradeep shankar³, Vijay Samueal Raj \\ ${ }^{1}$ Department of Physiotherapy, JSS College of Physiotherapy, Mysore, Karnataka, India. ${ }^{2}$ Department of Pulmonology, \\ JSS Medical College (JSSAHER), Mysore, Karnataka, India. ${ }^{3}$ Department of Physiotherapy, Medeor Hospital, Abu Dhabi. \\ ${ }^{4}$ Department of Physiotherapy, JSS College of Physiotherapy, Mysore, Karnataka, India.
}

\section{ABSTRACT}

\section{BACKGROUND}

It is well documented that exercise training for COPD patients improve the quality of life, and decrease hospitalization and therefore decreased the mortality. Exercise implementation may be influenced by many factors and it varies considerably between countries. There is an acute need to understand the factors that predict non-adherence and adherence to PR with a view to improve uptake. We wanted to determine the percentage of adherence to 12 weeks of exercise training program and to know the reason for non-adherence and the factors that motivated them to adhere to the exercise rehabilitation program.

\section{METHODS}

One hundred and thirty-four patients with COPD referred for rehabilitation participated in this study. The interview was conducted to the subjects dropped from the training and to subjects adhered to the intervention. Wide range of openended questions as well as probing questions, such as why they had decided to attend or not attend the exercise training program, and why they had decided to continue or drop out were asked. Interviews were carried out and audio-taped with the consent of the participant. The reasons for adherence and non-adherence were listed and analysed using descriptive statistics.

\section{RESULTS}

The COPD patients' adherence to three month of exercise training was $44.7 \%$ and drop out was $55.22 \%$. The reason for adherence to the exercise regimen to end was the influence of the referring doctor (15\%), understood the benefits and selfmotivated $(31.66 \%)$, positively influenced by other patients attending the exercise rehabilitation therefore motivated $(26.66 \%)$ and increased self-confidence $(26.66 \%)$. The reason for non-adherence for both the type of intervention was not affordable (29.72\%), no attender (family support) to accompany (13.51\%), LTOT use $(9.45 \%)$, breathlessness and leg fatigue $(10.81 \%)$, absence of therapist $(6.75 \%)$, travelling distance (6.75\%), hospitalizations (18.91\%), felt that they can do at home (2.70\%), shifting/migration (1.35\%).

\section{CONCLUSIONS}

Adherence of COPD patients to exercise rehabilitation program for twelve weeks is moderate.

\section{KEY WORDS}

Adherence, COPD, Pulmonary Rehabilitation, Exercise Training, Factors Predict, Non-Adherence
Corresponding Author:

Dr. Chaya $S . K$.,

Department of Pulmonology,

JSS Medical College (JSSAHER),

Mysore, Karnataka, India.

E-mail: chaya.sindaghatta@gmail.com

DOI: $10.14260 /$ jemds/2019/743

Financial or Other Competing Interests: None.

How to Cite This Article:

Mahadevan R, Chaya SK, Shankar $P$, et al. Adherence to exercise training in COPD patients- factors that predict patient adherence and non-adherence- a preliminary study. J. Evolution Med. Dent. Sci. 2019;8(46):3427-3431, DOI: $10.14260 /$ jemds $/ 2019 / 743$

Submission 14-09-2019,

Peer Review 31-10-2019,

Acceptance 06-11-2019,

Published 18-11-2019. 


\section{BACKGROUND}

COPD is currently the fourth leading cause of death in the world, but is projected to be the $3^{\text {rd }}$ leading cause of death by 2030.1 COPD represents an important public health challenge that is both preventable and treatable. COPD rates are expected to continue to rise steeply with an ever-increasing mortality rate. It is clear that the efforts to improve the health of those living with COPD need closer attention. Rigorous existing estimates of the general prevalence of chronic bronchitis in rural areas of India to lie between $6.5 \%$ and 7.7\%. COPD being a chronic progressive disease poses a huge economic burden on the patient as well as the health-care systems. At an individual level, it frequently proves to be financially ruin-some for families with average income. COPD is also one of the most common reasons for sick leave from work, placing an enormous and increasing economic burden on the society. ${ }^{2}$ The prevalence of COPD is related to age and smoking but is found to be underestimated due to unawareness of the disease in subjects suffering from COPD. In addition, a lack of correct diagnostics in those seeking medical advice is frequent. ${ }^{3}$ In response to the rising morbidity and mortality associated with this disease, a pulmonary rehabilitation program was developed to optimize the prevention and management of COPD. ${ }^{4}$ Pulmonary rehabilitation (PR) is a multidisciplinary approach to optimizing the physical and social functioning as well as the autonomy of the patients. PR offers the best chance to manage the symptoms and reduce health resource use.5,6 Recent research indicates that PR programs increase health related quality of life in patients with COPD.7,8 Nevertheless, available PR programs remain underused by COPD patients. ${ }^{9}$ The component of pulmonary rehabilitation is drugs, nutrition, oxygen therapy, education, psychological counseling, family members, exercise training and managing breathlessness. The well documented outcomes of exercise training are improved 6MWT (Functional capacity), improvement in dyspnoea (MMRC) and improved quality of life (SGRQ). ${ }^{9}$

Adherence to therapeutic interventions, including a healthy lifestyle and regular exercise programme, are crucial health behaviour in the management of chronic respiratory disease. Most of the studies have focused on the short-term benefits of pulmonary rehabilitation ${ }^{10,11}$ without addressing the challenges of long-term adherence to exercise. Exercise self-efficacy and expected benefits from regular exercise have been shown to be predictors of exercise adherence while depression is associated with poor adherence. In a review of 27 cross sectional and 14 longitudinal studies of individuals 65 years or older, educational level and past exercise behaviour were positively associated with regular exercise. ${ }^{12}$ Conversely, perceiving one's health as "poor" was the biggest barrier to exercise adoption and maintenance. Similar results were reported in a qualitative study of patients with COPD ${ }^{13}$ where barriers to lifestyle change included progression of COPD and associated comorbid conditions. A recent study of patients with COPD following pulmonary rehabilitation reported the most consistent barriers to adherence were chest infection and disease exacerbation. Few studies investigated the adherence by COPD patients to PR programs. Findings reveal a lack of perceived benefit from the PR program and inconvenient transport were significant concerns for patients for not being adhered to the programme. ${ }^{14}$ Other researchers identified factors from COPD patients concerning PR attendance and completion, such as group support, self-confidence, the influence of the referring physician,15 fear of being breathless and exacerbation of existing medical problems. ${ }^{16}$ Of note, while only a few studies explore patients' perspectives, no literature was found that explored perceptions of PR attendance from the health professionals' perspective working in PR programs. Given the lack of qualitative description regarding the experience of COPD patients following a PR program and the perspective of professional clinical staff, this study aimed to explore patients' perceptions of the attendance and completion of PR, within the context of outpatient PR programs. The overall goal was to better understand the experiences and thoughts attributed to PR attendance and to identify barriers and strategies to establish effective pulmonary rehabilitation.

The major goal of rehabilitation is to provide symptom relief, effective management of COPD which has shown to reduce the rate of exacerbations, hospitalizations, possibly mortality and to improve health related quality of life ${ }^{17}$. PR for COPD patients has been shown to be an effective nonpharmacological intervention; however uptake and completion of programmes is frequently low. ${ }^{18}$ Uptake for PR is poor with attendance reported as being as low as 50\%.In addition $23-31 \%$ of patients who start the course fail to complete. ${ }^{19}$ The cost, attitudes and beliefs for exercise training in PR varies considerably between countries. Exercise training is one of the components of pulmonary rehabilitation. It is well documented that exercise training for COPD patients improve the quality of life, and decrease hospitalization and therefore decreased the mortality. ${ }^{20}$ Exercise implementation may be influenced by local health system characteristics, program organization, and by individual patient characteristics varies considerably between countries. There is dearth on studies conducted on effect of pulmonary rehabilitation in developing countries like India therefore the adherence rate to the pulmonary rehabilitation is not assessed. There is an acute need to understand the factors that predict non-adherence and adherence to PR with a view to improve uptake. The purpose of this study is to know reason for non-adherence and the factors enhanced them to adhere to the exercise rehabilitation program.

\section{METHODS}

One hundred and thirty-four patients with COPD who were referred for rehabilitation participated in this study. The sample was time-bound where the data was collected in two years of period from patients enrolled to exercise training. Qualitative study using semi-structured interviews was conducted for COPD patients aged 45-70 years, referred for pulmonary rehabilitation over a 2-year period. The subjects included were COPD more than five years. There were two method of intervention carried for two different groups, conventional continuous training group and another was high intensity interval training group. The conventional continuous training is the training given in submaximal intensity (Modified Rate of Perceived Exertion rating 3-4) 
without interval until the patient able to perform. High intensity interval training is the training given at high intensity (Modified Rate of Perceived Exertion rating 3-4) with interval at 1 ; ratio ( $30 \mathrm{sec}$ high intensity, $30 \mathrm{sec}$ rest). The intervention was for 12 weeks. The mode of training was treadmill aerobic training. The frequency was 3 days in a week for up to 36 weeks. Attendance of more than $75 \%$ is considered as adherent to the exercise training. The patients were given education pamphlets and they watched educational video on managing breathlessness and importance and benefits of pulmonary rehabilitation once a week. The interview was conducted to the subjects dropped from the training and to subjects adhered to the intervention. Wide range of open-ended questions as well as probing questions, such as why they had decided to attend or not attend the exercise training program, and why they had decided to continue or drop out were asked. The questions like "what are the reasons that lead them to adhere to the exercise training" were asked to the patients who had adhered and had more than $70 \%$ attendance. Questions like "what are the reason that lead them for not adhering to the exercise training" were asked for patients who had less than $70 \%$ of adherence. Interviews were carried out and audiotaped with the consent of the participant.

\section{Statistical Analysis}

Descriptive analysis was used to analyse the adherence and non-adherence. Using percentage of attendance logbook. Bar graph is used to describe the percentage of adherence (Figure 2) and pie chart is used to describe the factors predicted the adherence and non-adherence to three months of exercise training by COPD patient (Figure 3 and 4).

\section{RESULTS}
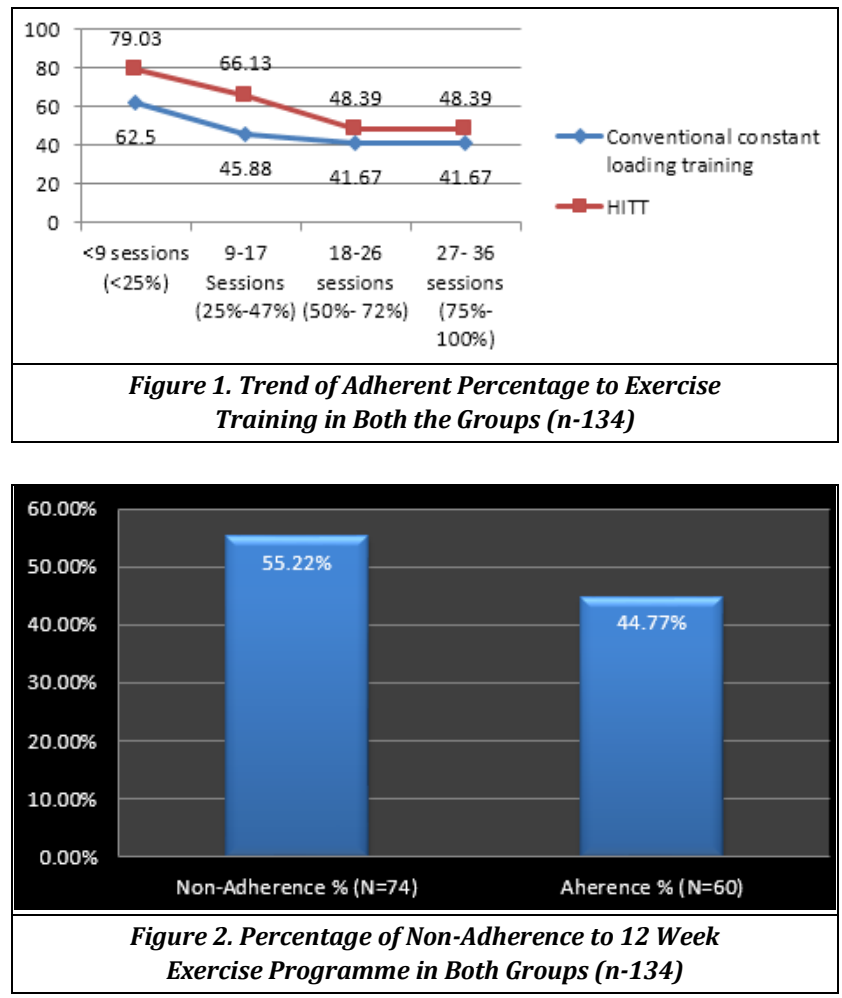
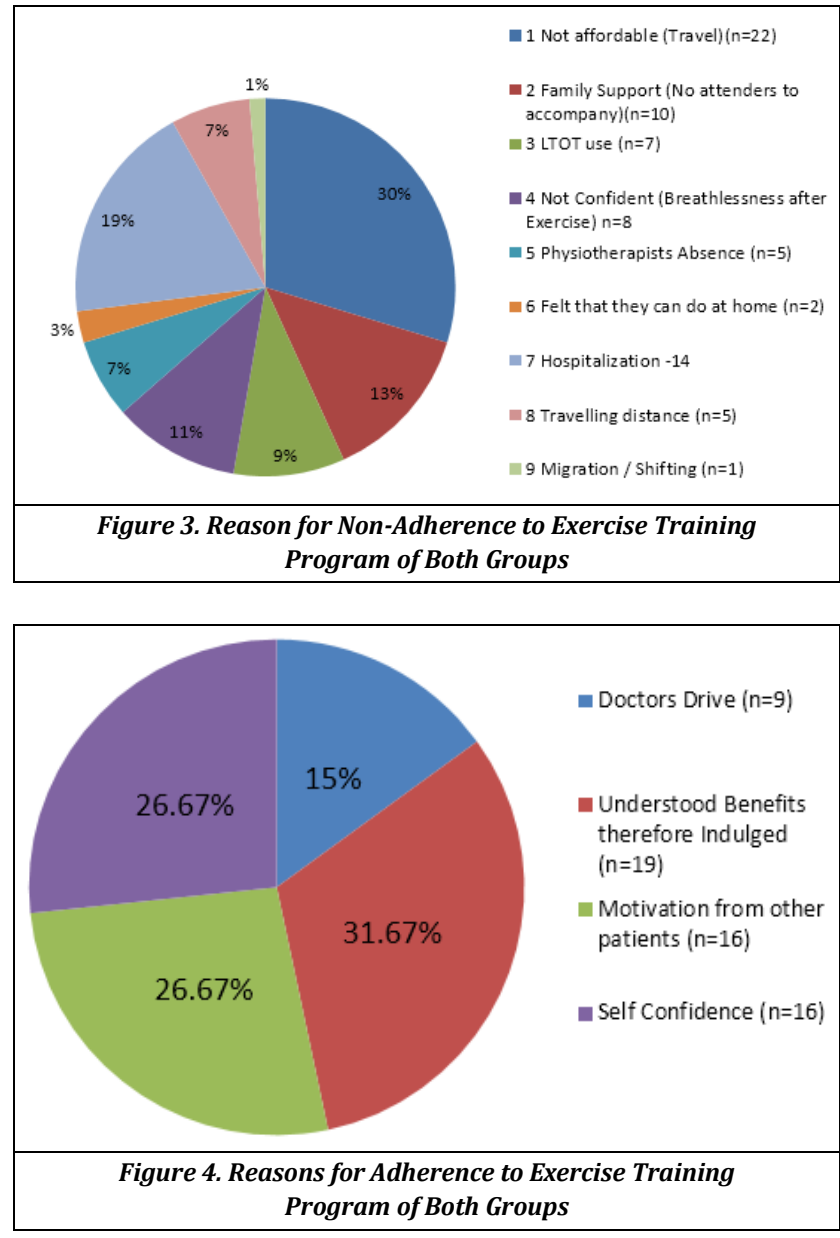

One hundred and thirty-four patients included in the study. The adherence to exercise for both conventional constant load training and HITT was $44.7 \%$ and drop out was $55.22 \%$. The adherence to conventional constant exercise training was (30) $41.6 \%$ and the drop out was $58.3 \%$. The adherence to high intensity interval training was $48.3 \%$ and the drop out was $51.6 \%$. The reason for non-adherence for both the type of intervention was not affordable (29.72\%), no attender (Family support) to accompany $(13.51 \%)$, LTOT use $(9.45 \%)$, breathless and leg fatigue $(10.81 \%)$, absent of therapist (6.75\%), travelling distance (6.75\%), hospitalizations $(18.91 \%)$, felt that they can do at home (2.70\%), shifting/migration (1.35\%). The adherence to the programme was positively influenced by other patients attending the exercise rehabilitation, and increased selfconfidence Twenty five percent were accountable to factors beyond patients' control having medical reasons like exacerbations/ hospitalizations and absent therapists.

The reason for adherence to the exercise regimen to end was the influence of the referring doctor (15\%), understood the benefits and self-motivated (31.66\%), positively influenced by other patients attending the exercise rehabilitation therefore motivated $(26.66 \%)$ and increased Self-confidence (26.66\%).

\section{DISCUSSION}

Strategy to enhance adherence will not only improve patient health outcomes but also the health status of patients and 
reduce the economic and societal burden associated with COPD. The adherence to exercise training of both conventional constant load training and HITT was $44.7 \%$ and drop out was $55.22 \%$. The adherence to conventional constant exercise training was $41.6 \%$ and the drop out was $58.3 \%$. For both groups, the adherence rate is moderate. The reason for non-adherence is predominantly due to unaffordability, hospitalization, less family support, breathless with leg fatigue and travel distance. Most of the patients enrolled were from rural area and they even had problem to afford for medication had to also bare travel cost which led to dropout. In a study it was found that patients travelled for less than seven minutes were likely to remain in the exercise training program than patients travelling for longer periods of time. ${ }^{21}$ In this study the patient dropped as most of them were travelling more than 30 minutes.

In recent studies the patients with COPD following pulmonary rehabilitation reported the most consistent barriers to adherence were chest infection and disease exacerbation or hospitalization, ${ }^{22}$ likewise in this study $25 \%$ of patients not adhered to exercise regimen was due to the same reason. The reason for exacerbation can be lack of knowledge about the disease management. The reason for non-adherence due to breathless and leg fatigue is reported in conventional constant load training as interval training had rest period and therefore no report on episodes of leg fatigue and breathlessness which led to anxiety is reported. The factors for adherence to exercise training program reported by the patients were predominantly due to influenced by other patients attending the exercise rehabilitation and selfconfidence therefore the implementation of buddy system may influence this factor which in turn increase the adherence rate.

The reason for non-adherence was due to unaffordability, less family support, and travel distance, because most of the patient were from rural area. Most of the patients were old and many had to travel more distance with oxygen support which demanded family support. Patients travelled less than seven minute, adhered to exercise training. ${ }^{23}$ The reason for non-adherence due to breathless and leg fatigue is reported from the patients whose grades of disease was severe to very severe. In this study $25 \%$ of patients not adhered to exercise regimen was Hospitalization/Acute exacerbation and absent of therapist or change in schedule.

The factors for adherence were predominantly due to influence of other patients attending the exercise rehabilitation and self-confidence. The aspect of enjoyment, increased confidence and self-esteem could be emphasized in addition to the physical benefits of attending PR. ${ }^{24}$ Fraser and Spink, reported that the cohesion shown by the group, were important determinant. ${ }^{25}$ In this study $27 \%$ of the patients adhered as they met other patients which had motivated them for participation. The referring doctor was found to have a role in influencing the uptake of PR. In this study 15\% of them adhered due to doctors' reference.

To enhance uptake and completion of pulmonary rehabilitation programs, more attention is required to transportation. Simple, cost-effective approaches may encourage more patients with COPD to participate in a therapeutic intervention. Recent strong evidence base Implementation is buddy system which may influence the patients to adhere to the exercise training, which in turn increase the adherence rate. The referring doctor was found to have a role in influencing the uptake of PR. It should be possible to develop a strategic package aimed at helping referring doctors to promote adherence. Strategy to enhance adherence will not only improve patient health outcomes but also the health status of patients and reduce the economic and societal burden associated with COPD.

\section{CONCLUSIONS}

The adherence of COPD patients to exercise rehabilitation program for twelve weeks is moderate. The factors predominantly that led to non-adherence was unaffordability, less family support, breathlessness, leg fatigue and less predominantly was travelling distance, migration/ shifting, patients' belief that they can manage to do exercise at home itself, hospital admission/ exacerbation, absence of therapist or change in the schedule. The factors led to adherence were, influenced by other patients attending the exercise rehabilitation, self-confidence, understanding the benefits of training and influence of the referred doctor. These factors can be considered to implement comprehensive rehabilitation services which include exercise intervention as one of the components in COPD patients. Considering these factors may improve the adherence rate and benefit the COPD patients.

\section{REFERENCES}

[1] Lozano R, Naghavi M, Foreman K, et al. Global and regional mortality from 235 causes of death for 20 age groups in 1990 and 2010: a systematic analysis for the Global Burden of Disease Study 2010. Lancet 2012;380(9859):2095-128.

[2] Mathers CD, Loncar D. Projections of global mortality and burden of disease from 2002 to 2030. PLoS Med 2006;3(11):e442.

[3] Indian Council of Medical Research Task Force Study (1993-98). Project Report - Estimation of costs of management of smoking related chronic obstructive pulmonary disease and coronary heart disease.

[4] Ries AL, Bauldoff GS, Carlin BW, et al. Pulmonary rehabilitation: Joint ACCP/AACVPR evidence-based clinical practice guidelines. Chest 2007;131(Suppl 5):4S$42 \mathrm{~S}$.

[5] Rubi M, Renom F, Ramis F, et al. Effectiveness of pulmonary rehabilitation in reducing health resources use in chronic obstructive pulmonary disease. Arch Phys Med Rehabil 2010;91(3):364-8.

[6] Corhay JL, Dang DN, Van Cauwenberge H, et al. Pulmonary rehabilitation and COPD: providing patients a good environment for optimizing therapy. Int J Chron Obstruct Pulmon Dis 2014;9:27-39.

[7] Bentsen SB, Rokne B, Wahl AK. Comparison of healthrelated quality of life between patients with chronic obstructive pulmonary disease and the general population. Scand J Caring Sci 2013;27(4):905-12. 
[8] Puhan MA, Lareau SC. Evidence-based outcomes from pulmonary rehabilitation in the chronic obstructive pulmonary disease patients. Clin Chest Med 2014;35(2):295-301.

[9] Johnston K, Grimmer-Somers K. Pulmonary rehabilitation: overwhelming evidence but lost in translation? Physiother Can 2010;62(4):368-73.

[10] Lacasse Y, Wong E, Guyatt GH, et al. Meta-analysis of respiratory rehabilitation in chronic obstructive pulmonary disease. Lancet 1996;348(9035):1115-9.

[11] Maspero JF, Duenas-Meza E, Volovitz B, et al. Oral montelukast versus inhaled beclomethasone in 6- to 11year-old children with asthma: results of an open-label extension study evaluating longterm safety, satisfaction, and adherence with therapy. Curr Med Res Opin 2001;17(2):96-104.

[12] McAuley E, Lox C, Duncan TE. Long-term maintenance of exercise, self-efficacy, and physiological change in older adults. J Gerontol 1993;48(4):P218-P24.

[13] Nault D, Dagenais J, Perreault V, et al. Qualitative evaluation of a disease specific self-management program "Living well with COPD". Eur Respir J 2000;16:317S.

[14] Keating A, Lee AL, Holland AE. Lack of perceived benefit and inadequate transport influence uptake and completion of pulmonary rehabilitation in people with chronic obstructive pulmonary disease: a qualitative study. J Physiother 2011;57(3):183-90.

[15] Aronld E, Bruton A, Ellis-Hill C. Adherence to pulmonary rehabilitation: a qualitative study. Resp Med 2006;100(10):1716-23.

[16] Harris D, Hayter M, Allender S. Improving the uptake of pulmonary rehabilitation in patients with COPD: qualitative of the experiences and attitudes. $\mathrm{Br} \mathrm{J}$ of Gen Pract 2008;58(555):703-10.

[17] Sin DD, McAlister FA, Man SF, et al. Contemporary management of chronic obstructive pulmonary disease: scientific review. JAMA 2003;290(17):2301-12.

[18] Chapman KR, Mannino DM, Soriano JB, et al. Epidemiology and costs of chronic obstructive pulmonary disease. Eur Respir J 2006;27(1):188-207.

[19] Keating A, Lee A, Holland AE. What prevents people with chronic obstructive pulmonary disease from attending pulmonary rehabilitation? A systematic review. Chron Respir Dis 2011;8(2):89-99.

[20] Fischer MJ, Scharloo M, Abbink JJ, et al. Drop-out and attendance in pulmonary rehabilitation: the role of clinical and psychosocial variables. Respir Med 2009;103(10):1564-71.

[21] Garrod R, Marshall J, Barley E, et al. Predictors of success and failure in pulmonary rehabilitation. Eur Respir J 2006;27(4):788-94.

[22] Hayton C, Clark A, Olive S, et al. Barriers to pulmonary rehabilitation: characteristics that predict patient attendance and adherence. Respiratory Medicine 2013;107(3):401-7.

[23] Brooks D, Krip B, Mangovski-Alzamora S, et al. The effect of postrehabilitation programmes among individuals with chronic obstructive pulmonary disease. Eur Respir J 2002;20(1):20-9.

[24] McAuley E, Blissmer B. Self-efficacy determinants and consequences of physical activity. Exerc Sport Sci Rev 2000;28(2):85-8.

[25] Fraser SN, Spink KS. Examining the role of social support and group cohesion in exercise compliance. J Behav Med 2002;25(3):233-49. 